\title{
Energy Efficiency and SINR Maximization Beamformers for Spectrum Sharing with Sensing Information
}

\author{
AbdulRahman Alabbasi, Student Member, IEEE, Zouheir Rezki, Senior Member, IEEE, Basem Shihada, Senior \\ Member, IEEE, CEMSE Division, King Abdullah University of Science and Technology \\ Email: \{abdulrahman.alabbasi, zouheir.rezki, basem.shihada\}@kaust.edu.sa
}

\begin{abstract}
In this paper we consider a cognitive radio multiinput multi-output environment in which we adapt our beamformer to maximize both energy efficiency (EE) and signal to interference plus noise ratio (SINR) metrics. Our design considers an underlaying communication using adaptive beamforming schemes combined with the sensing information to achieve optimal energy efficient systems. The proposed schemes maximize EE and SINR metrics subject to cognitive radio and quality of service constraints. The analysis of the proposed schemes is classified into two categories based on the knowledge of the secondary transmitter to primary receiver channel. Since the optimization of EE and SINR problems are not convex problems, we transform them into a standard semi-definite programming (SDP) form to guarantee that the optimal solutions are global. Analytical solution is provided for one scheme, while the second scheme is left in a standard SDP form. Selected numerical results are used to quantify the impact of the sensing information on the proposed schemes compared to the benchmark ones.
\end{abstract}

Index Terms-Cognitive radio, spectrum sharing, energy efficiency, spectrum sensing, resource allocation, beamforming, MIMO.

\section{INTRODUCTION}

Green communication is a major contributor to the global greenness. Therefore, we note the high demend on reducing the mobile phones power consumption while achieving high throughput. It is found that the radio's power consumption reaches up to 50 percent of the mobile device's battery power consumption [1]. One of the green communication potential candidates is cognitive radio (CR) systems [2]. There are many proposals to achieve greenness for CR technology [3], [4], [5],[6]. CR has been proposed to overcome the inefficient use of frequency spectrum and its resulting scarcity. In CR, secondary users (SU) may share the bands of the primary users (PU) through various schemes [7], [8]. Combining the knowledge of PU's existence into the SU's transmission scheme results in an improved performance. Therefore, existing work utilizes the PU's sensing information in single-input singleoutput systems [9].

Researchers have been considering optimal energy efficient systems by minimizing the system energy while preserving its quality of service (QoS) parameters, such as rate, delay, etc. [10], [11], [12]. However, it has been indicated in [13], [14], [15] that optimizing the energy per goodbits (EPG) yields to an improved result in terms of energy efficiency comparing to both minimizing the system energy, subject to QoS and power constraints, and maximizing the rate, subject to power constraint. This results have been supported in our numerical result section. Authors in [16] have tackled the problem of maximizing the energy efficiency (EE) metric, which is the inverse of EPG, in multi-input multi-output (MIMO) multiuser environment.

Recent development of MIMO beamfroming techniques creates a new dimension for co-channel users to coexist without causing severe interference to each others [17], [18], [19]. In conventional methods, beamforming is obtained by either maximizing the signal to interference plus noise ratio (SINR), or minimizing the transmission energy subject to a minimum SINR constraint. Authors in [20] formulate the SU beamforming problem as a non-convex optimization problem, under the assumption that the CSI on all links is perfectly known to the SUs. Additionally, authors in [21] considered the opportunistic communication, as they exploit multi-antennas at the secondary transmitter to effectively balance between spatial multiplexing for the secondary transmitter and interference avoidance at the primary receiver. They used algorithms based on singular-value decomposition (SVD) of the SU MIMO channel.

In this paper, we maximize EE and SINR metrics under spectrum sharing MIMO environment utilizing sensing information about PU. The SU's SINR maximization scheme (scheme 1) is constrained with a certain peak power and interference threshold towards the PU. The SU's EE maximization scheme (scheme 2) is constrained with a minimum rate constraint in addition to the constraints stated for scheme 1. While analyzing the above proposed systems, we consider two scenarios of knowledge about the secondary to primary user's channel. In the first scenario, the SU has no knowledge of the secondary transmitter to primary receiver channel. While in the second scenario, the SU have instantaneous values of the secondary to primary channel information. Note that, as in the second scenario, many work considered the availability of PU perfect or partial CSI or PU signal information through a CR engine or manager [22],[23]. We assume the availability of a spectrum sensor at the SU. This sensor periodically collects real-time information about PU signals in the surrounding environment. This real-time information assists SU to aggregate the maximum power (using the adaptive beamforming vectors) within the tolerable interference threshold (set by the communication organizations). 
Unlike the work in [16], which analyzes the EE metric without considering a PU protection constraint and no utilization of the sensing information, our work protects the PU and utilizes the sensing information. Moreover, authors in [24] did not consider the EE metric in their analysis which makes our system unique in many ways.

Our solution methodology is divided into two parts. In the first part, we introduce the sensing scheme analysis and its impact on the optimal beamformers. The secondary sensor utilizes a blind multi-antenna combining scheme, because we assume no information about the primary transmitter to the sensor channel. In order to characterize the sensor performance and its impact on both maximization problems we have provided analytical expressions for the probability of error performance. In the second part, we tackle distinct optimization techniques used to solve both maximization problems. The problem of scenario 1 in scheme 1 is solved by transforming it into an eigenvalue problem. On the other hand, we transform the problem of scheme 2 into a standard semidefinite programming (SDP) problem to guarantee a global optimal solution. An analytical result is provided for scheme 1 , whereas, the problem of scheme 2 and the second scenario of scheme 1 are written in a standard SDP form, which can be solved in a polynomial time [25]. Numerical results quantify the improvement gain obtained by the proposed schemes compared to the benchmark ones. Furthermore, we quantify the performance difference between the optimal beamformer which maximizes scheme 1 and the optimal beamformer which maximizes scheme 2 from an energy efficiency perspective.

This paper is organized as follows. Section II describes the system model with some related background on PU blind multi-antenna sensing approach. Section III presents the common constraints and preliminaries for both targeted optimization problems. Section IV tackles the problem of maximizing SINR metric subject to peak power and interference constraints. In section $\mathrm{V}$ we maximize the EE metric subject to minimum rate, peak power, and interference constraints. Finally, some numerical results are presented in section VI.

\section{System MOdEL AND RELATED BACKGROUND}

\section{A. System Model}

In this system, we consider a CR environment under MIMO channels. Our design aims to achieve an energy efficient system that utilizes the sensing information through an adaptive beamforming schemes. We consider the sensing information effect on the following cases,

1) Maximizing the SU's SINR under both a peak power constraint and interference constraints.

2) Maximizing the SU's EE metric (defined in section V) under peak power, interference, and minimum rate constraints.

Figure 1 shows the system model under the assumption of MIMO channels for ST-SR, PT-SR, and ST-PR, where ST and $\mathrm{SR}$ are the secondary transmitter and receiver, respectively. PT and PR are the primary transmitter and receiver, respectively. The SU sensor assumed to be located at the SR, while the sensing information is exchanged between SR and ST through a low-rate error free feedback channel without considerable delay. We assume that ST has $N_{t}$ antennas, SR has $N_{r}$ antennas, PT has $M_{t}$ antennas, and PR has $M_{r}$ antennas. The channels depicted in Fig. 1 are as follows, $\mathbf{H}_{p s} \in \mathbb{C}^{N_{r} \times M_{t}}$ is the channel between PT-SR, $\mathbf{H}_{s p} \in \mathbb{C}^{M_{r} \times N_{t}}$ is the channel between ST-PR, and $\mathbf{H}_{s} \in \mathbb{C}^{N_{r} \times N_{t}}$ is the channel between SR and ST. These channels are independent of each other and are identically distributed (i.i.d), with entries of each matrix following a circularly symmetric complex Gaussian random variables with zero mean and unit variance. The CSI of $\mathbf{H}_{s}$ is assumed to be known and shared between ST and SR through the feedback channel. Two scenarios are considered for the CSI of $\mathbf{H}_{s p}$ as follows,

- Scenario 1: No knowledge about neither $\mathbf{H}_{s p}$ nor $\mathbf{H}_{p s}$ at both ST and SR.

- Scenario 2: Perfect knowledge of $\mathbf{H}_{s p}$ and $\mathbf{H}_{p s}$ shared between SR and ST. Note that we do not utilize the knowledge of $\mathbf{H}_{p s}$ neither at the sensing scheme nor at the communication scheme. The interference from PU through $\mathbf{H}_{p s}$ is decoded at $\mathrm{SR}$ as noise without any successive interference cancelation scheme.

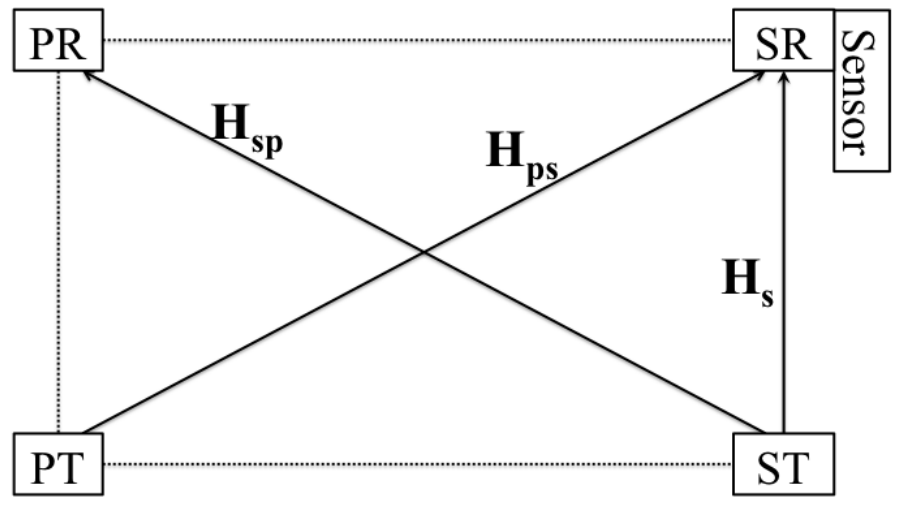

Fig. 1. System Model.

\section{B. Sensing Background}

In this section, we outline the sensing approach used in the proposed system. This approach is based on blind combining the primary signal at the secondary sensor, since no knowledge of $\mathbf{H}_{p s}$ is utilized. Then, we apply the energy detector to the output of the blind combiner. This sensing scheme have appeared in [26], we recall it here for self-contentedness of the paper. However, we note that the analytical results derived here have not been reported in [26]. These results characterize the sensor error performance, and thereby allow obtaining the interference threshold of the beam forming weights. The reason behind adopting a blind combining scheme is the assumption of not having prior knowledge about PU signal, $\mathbf{H}_{p s}$ and $\mathbf{H}_{p s}$ channels information, as in scenario 1. We begin the description of the blind combining sensing scheme by explaining how to obtain the combining weights of the multiantenna SU sensor. Then we present the analytical results by describing the PDF of the blind sensor output.

When the primary user is transmitting, the SU sensor observes a signal $\mathbf{Y}=\left[\mathbf{y}_{1}, \mathbf{y}_{2}, \ldots, \mathbf{y}_{N_{s}}\right]$, with independent 
complex Gaussian columns, each expressed as $\mathbf{y}_{i} \in \mathbb{C}^{N_{r} \times 1}$, $N_{s}$ is the number of sensing samples. The received vector $\mathbf{y}_{i}$ is given by,

$$
\mathbf{y}_{i}=\mathbf{H}_{p s, i} \mathbf{x}_{p, i}+\mathbf{n}_{s, i} \quad i=1, \ldots, N_{s}
$$

where $i$ is the sampling index, $\mathbf{x}_{p, i}$ is the PT transmitted signal at sample $i$, the vector $\mathbf{n}_{s, i}$ represents an additive white Gaussian noise (AWGN) with zero mean and variance $\frac{N_{0}}{2}$ per dimension at SR. It is noted that $\mathbf{x}_{p, i}$ is independent of $\mathbf{H}_{p s, i}$, since the primary transmitter does not know about the channel to the secondary receiver. The column vector $\mathbf{y}_{i}$ has a zero mean and covariance ma$\operatorname{trix} \boldsymbol{\Sigma}=\mathbb{E}_{\mathbf{x}_{p, i}, \mathbf{H}_{p s, i}}\left\{\left(\mathbf{H}_{p s, i} \mathbf{x}_{p, i}+\mathbf{n}_{s, i}\right)\left(\mathbf{H}_{p s, i} \mathbf{x}_{p, i}+\mathbf{n}_{s, i}\right)^{\dagger}\right\}$, where $\mathbb{E}\{$.$\} is the expectation operator. Since we consider$ I.I.D. Rayleigh fading central channel, then the covariance matrix is expressed as $\boldsymbol{\Sigma}=\sigma \mathbf{I}$, where $\sigma$ is the variance of the received primary user signal at secondary user sensor. The SU sensor then computes the normalized Wishart matrix,

$$
\mathbf{R}_{\mathbf{y}}=\boldsymbol{\Sigma}^{-1} \mathbf{Y} \mathbf{Y}^{\dagger}=\frac{1}{\sigma} \mathbf{Y} \mathbf{Y}^{\dagger},
$$

where the upper script $(.)^{\dagger}$ designates the conjugate transpose of its argument.

Using the Eigen-Value-Decomposition (EVD) method, the following statement is true,

$$
\mathbf{R}_{\mathbf{y}} \mathbf{V}=\mathbf{V} \boldsymbol{\Lambda},
$$

where

$$
\boldsymbol{\Lambda}=\left[\begin{array}{cccc}
v_{r} & \cdot & \cdot & \cdot \\
\cdot & \ddots & \cdot & \cdot \\
\cdot & \cdot & v_{2} & \cdot \\
\cdot & \cdot & \cdot & v_{1}
\end{array}\right], \mathbf{V}=\left[\begin{array}{lll}
\mathbf{v}_{\mathbf{r}} & \ldots & \mathbf{v}_{\mathbf{1}}
\end{array}\right] .
$$

where $r=\min \left(N_{r}, N_{s}\right)$. In (4), $v_{r} \geq \ldots \geq v_{2} \geq v_{1}$ are the Wishart matrix's eigenvalues. The vectors $\mathbf{v}_{\mathbf{r}}, \ldots, \mathbf{v}_{\mathbf{1}}$ are the corresponding eigenvectors. To realize the maximum SNR, we use the eigenvector that corresponds to the largest eigenvalue as the combining weight $\mathbf{w}$ for the SU sensor antennas, $\mathbf{w}=$ $\mathbf{v}_{\mathbf{r}}$. The output of the combiner is expressed as:

$$
\mathbf{y}_{\text {evd }}=\mathbf{w}^{\dagger} \mathbf{Y} \text {. }
$$

where $\mathbf{y}_{\text {evd }} \in \mathbb{C}^{1 \mathrm{x} N_{s}}$. Note that,

$$
\begin{aligned}
& \mathbf{y}_{e v d} \mathbf{y}_{e v d}^{\dagger}=\mathbf{w}^{\dagger} \mathbf{Y} \mathbf{Y}^{\dagger} \mathbf{w}=\sigma \mathbf{w}^{\dagger} \mathbf{R}_{\mathbf{y}} \mathbf{w}=\sigma \mathbf{v}_{\mathbf{r}}^{\dagger} v_{r} \mathbf{v}_{\mathbf{r}} \\
& =\sigma v_{r}\left\|\mathbf{v}_{\mathbf{r}}\right\|^{2} \stackrel{(a)}{=} \sigma v_{r}
\end{aligned}
$$

where (a) is obtained because the norm of the combining weights $\left\|\mathbf{v}_{\mathbf{r}}\right\|$ is unity. This confirms the fact that the EVD scheme uses combining weights that beamforms the sensing antennas to the largest eigenmode of the SR-PT channel. We apply the conventional energy detector to the combiner output signal $\mathbf{y}_{\text {evd }}$. The output of the energy detector before decision making step is as follows,

$$
\zeta\left(N_{s}\right)=\frac{1}{N_{s}} \sum_{i=1}^{N_{s}}\left|\mathbf{y}_{e v d}(i)\right|^{2}=\frac{1}{N_{s}} \mathbf{y}_{e v d} \mathbf{y}_{e v d}^{\dagger}=\frac{\sigma}{N_{s}} v_{r} .
$$

It follows that the distribution of $\zeta\left(N_{s}\right)$ is (up to a scaling) similar to the largest eigenvalue distribution [27] with a probability density function (PDF) as follows,

$$
f\left(v_{r}\right)=\frac{\left|\mathbf{\Psi}_{c}\left(v_{r}\right)\right| \operatorname{tr}\left(\mathbf{\Psi}_{c}^{-1}\left(v_{r}\right) \boldsymbol{\Phi}_{c}\left(v_{r}\right)\right) U\left(v_{r}\right)}{\prod_{k=1}^{r} \Gamma(t-k+1) \Gamma(r-k+1)},
$$

where $r=\min \left(N_{r}, N_{s}\right)$ and $t=\max \left(N_{r}, N_{s}\right)$, the function $U($.$) is a unit step function, note that the output of the unit step$ is one if the input is positive. The function $\Gamma($.$) is the gamma$ function [28, Sec.8.31], the function $\boldsymbol{\Phi}_{c}\left(v_{r}\right)$ is an $r \times r$ matrix whose entries are given by, $\left[\boldsymbol{\Phi}_{c}\left(v_{r}\right)\right]_{i, j}=v_{r}^{t-r+i+j-2} e^{-v_{r}}$, and $\left[\Psi_{c}(x)\right]_{i, j}=\gamma(t-r+i+j-1, x), \forall i, j=1, \ldots, r$, where, the $\gamma($.$) is the incomplete gamma function and$ $\left|\boldsymbol{\Psi}_{c}\left(v_{r}\right)\right|$ is the determinant of $\boldsymbol{\Psi}_{c}\left(v_{r}\right)$.

Reformulating (7) in terms of PU existence such that,

$\zeta\left(N_{s}\right)= \begin{cases}\zeta_{1}\left(N_{s}\right)=\frac{1}{N_{s}} \sum_{i=1}^{N_{s}}\left|\mathbf{w}^{\dagger}\left[\mathbf{H}_{p s, i} \mathbf{x}_{p, i}+\mathbf{n}_{s, i}\right]\right|^{2} & ; \mathcal{H}_{1} \\ \zeta_{0}\left(N_{s}\right)=\frac{1}{N_{s}} \sum_{i=1}^{N_{s}}\left|\mathbf{w}^{\dagger} \mathbf{n}_{s, i}\right|^{2} & ; \mathcal{H}_{0}\end{cases}$

where $\mathcal{H}_{1}$ and $\mathcal{H}_{0}$ are the hypotheses at which the primary user is active and idle, respectively. It follows that $f_{1}\left(v_{r}\right)$ and $f_{0}\left(v_{r}\right)$ are the corresponding PDF of $\zeta_{1}\left(N_{s}\right)$ and $\zeta_{0}\left(N_{s}\right)$, respectively. Note that $f_{1}\left(v_{r}\right)$ and $f_{0}\left(v_{r}\right)$ have the same distribution as (8) with different parameters $v_{r}$ and $\sigma$. Based on the two hypotheses in (9) we divide the output of the SU sensor into four regions as follows,

$$
\begin{array}{lllll}
\mathcal{R}_{11} & : & \zeta\left(N_{s}\right) \geq \gamma_{t h} & \text { under } & \mathcal{H}_{1} \text { (Correct detection) } \\
\mathcal{R}_{10} & : & \zeta\left(N_{s}\right) \geq \gamma_{t h} & \text { under } & \mathcal{H}_{0} \text { (False alarm) } \\
\mathcal{R}_{01} & : \quad \zeta\left(N_{s}\right) \leq \gamma_{t h} & \text { under } & \mathcal{H}_{1} \text { (Miss detection) } \\
\mathcal{R}_{00} & : & \zeta\left(N_{s}\right) \leq \gamma_{t h} & \text { under } & \mathcal{H}_{0} \text { (Correct event) }
\end{array}
$$

where $\gamma_{t h}$ is the detection threshold. Let us note the probability of each region as, $\operatorname{Pr}\left\{\mathcal{R}_{11}\right\}=P_{D}, \operatorname{Pr}\left\{\mathcal{R}_{10}\right\}=P_{F A}$, $\operatorname{Pr}\left\{\mathcal{R}_{01}\right\}=P_{M D}=1-P_{D}$, and $\operatorname{Pr}\left\{\mathcal{R}_{00}\right\}=1-P_{F A}$. Our purpose of using the sensing is to link the power allocation scheme to the sensing results. In other word, based on the regions defined in (10) we define, in Section IV, different thresholds for the interference caused to the PR by the ST. In order to achieve more protection for the PR, we divide the regions in $(10)$ into two events. The first event (called $\mathcal{A}$ ) occurs when the SU sensor declare the existence of PU. We define the probability of event $\mathcal{A}$ as follows (knowing that $\left.\operatorname{Pr}\left\{\mathcal{H}_{1}\right\}+\operatorname{Pr}\left\{\mathcal{H}_{0}\right\}=1\right)$

$$
\begin{aligned}
& \operatorname{Pr}\{\mathcal{A}\}=\operatorname{Pr}\left\{\mathcal{R}_{11} \cup \mathcal{R}_{10}\right\}= \\
& \operatorname{Pr}\left\{\zeta\left(N_{s}\right) \geq \gamma_{t h} \mid \mathcal{H}_{1}\right\} \operatorname{Pr}\left\{\mathcal{H}_{1}\right\}+\operatorname{Pr}\left\{\zeta\left(N_{s}\right) \geq \gamma_{t h} \mid \mathcal{H}_{0}\right\} \operatorname{Pr}\left\{\mathcal{H}_{0}\right\} \\
& =\int_{\gamma_{t h}}^{\infty} f_{1}\left(v_{r}\right) d v_{r} \operatorname{Pr}\left\{\mathcal{H}_{1}\right\}+\int_{\gamma_{t h}}^{\infty} f_{0}\left(v_{r}\right) d v_{r} \operatorname{Pr}\left\{\mathcal{H}_{0}\right\} \\
& =1-\int_{0}^{\gamma_{t h}} f_{1}\left(v_{r}\right) d v_{r} \operatorname{Pr}\left\{\mathcal{H}_{1}\right\}-\int_{0}^{\gamma_{t h}} f_{0}\left(v_{r}\right) d v_{r} \operatorname{Pr}\left\{\mathcal{H}_{0}\right\} \\
& =1-F_{1}\left(v_{r}\right) \operatorname{Pr}\left\{\mathcal{H}_{1}\right\}-F_{0}\left(v_{r}\right) \operatorname{Pr}\left\{\mathcal{H}_{0}\right\},
\end{aligned}
$$

where the functions $F_{0}\left(v_{r}\right)$ and $F_{1}\left(v_{r}\right)$ are the corresponding cumulative density functions (CDF) under hypotheses $\mathcal{H}_{0}$ and $\mathcal{H}_{1}$, respectively [27]. Since $\mathcal{A}^{c}$ is the event where the $\mathrm{SU}$ sensor declares that $\mathrm{PU}$ is idle, then $\operatorname{Pr}\left\{\mathcal{A}^{c}\right\}=1-\operatorname{Pr}\{\mathcal{A}\}$.

It is known that in the second scenario, SU has knowledge about the secondary to primary channel, the optimal sensing 
scheme is not a blind sensing, e.g. matched filter can be used. However, to preserve a fair comparison between the first and the second scenarios, it is required to use the same sensing scheme for both of them. Moreover, a sensing scheme that utilizes full knowledge about secondary to primary CSI and PU signal cannot be implemented in the first scenario, no knowledge about secondary to primary CSI. Therefore, the best option is to use a blind sensing scheme for both scenarios, as shown above.

\section{Preliminaries \& COMmon Constraints}

In this section, we present some preliminaries and common constraints that are shared by the maximization of SINR and EE problems. The received signal at SR is expressed as [24],

$$
\mathbf{y}=\mathbf{H}_{p s} \mathbf{x}_{p}+\mathbf{H}_{s} \mathbf{u}_{s} x_{s}+\mathbf{n}_{s}
$$

where $\mathbf{y}=\left[y_{1}, \ldots, y_{N_{r}}\right]$. The vector $\mathbf{u}_{s}$ is the beamforming vector of the secondary transmitter, $x_{s}$ is a scaler that represents the secondary transmitted data, without loss of generality we consider $\mathbb{E}\left\{\left|x_{s}\right|^{2}\right\}=1$. Note that, $\left\|\mathbf{x}_{p}\right\|^{2}=P_{p}$ and $\left\|\mathbf{u}_{s}\right\|^{2} \leq P_{\max }$ are the transmission power for both PT and $\mathrm{ST}$, respectively. The received signal after multiplying it by the received beamforming vector is,

$$
r=\mathbf{v}_{s}^{\dagger} \mathbf{y}
$$

where $\mathbf{v}_{s}$ is the post-coding vector at SR, note that $\left\|\mathbf{v}_{s}\right\|^{2}=1$.

The first common constraint is the peak power constraint $\left(\mathfrak{C}_{1}\right)$ on the ST transmission power, which is expressed as follows,

$$
\mathfrak{C}_{1}:\left\|\mathbf{u}_{s}\right\|^{2} \leq P_{\max }
$$

where $P_{\max }$ is the maximum peak power limit on the secondary transmitter.

The second common constraint is the statistical interference constraint $\left(\mathfrak{C}_{2}\right)$ on the ST interference power affecting the PR, expressed as below,

$$
\mathfrak{C}_{2}: \operatorname{Pr}_{\mathbf{v}_{p}, \mathbf{H}_{s p}}\left\{\left|\mathbf{v}_{p}^{\dagger} \mathbf{H}_{s p} \mathbf{u}_{s}\right|^{2} \leq P\right\} \geq 1-\delta,
$$

where the vector $\mathbf{v}_{p}$ is the PR weighting vector, $\left\|\mathbf{v}_{p}\right\|^{2}=1$, the constant $P$ is the interference threshold toward the PR which is decided based on the sensing information, and $\delta$ is the probability value with which the ST is allowed to violate the interference threshold $P$.

\section{MAXIMIZING SINR}

In this section, we formulate the problem of maximizing SINR utilizing the sensing information.

Based on the received signal in (13), the corresponding secondary user SINR is [29], [24],

$$
\mathrm{SINR}=\frac{\left(\mathbf{v}_{s}^{\dagger} \mathbf{H}_{s} \mathbf{u}_{s}\right)^{\dagger}\left(\mathbf{v}_{s}^{\dagger} \mathbf{H}_{s} \mathbf{u}_{\mathbf{s}}\right)}{\left(\mathbf{v}_{s}^{\dagger} \mathbf{H}_{p s} \mathbf{x}_{p}\right)^{\dagger}\left(\mathbf{v}_{s}^{\dagger} \mathbf{H}_{p s} \mathbf{x}_{p}\right)+N_{0}}
$$

Note that our choice of this SINR formula follows from several facts. This expression is an achievable SINR, since there is interference from the PU and the MIMO system is not a point to point. Moreover, we consider a similar SINR formula in the EE maximization problem for the ease of transformation to an SDP problem. Therefore, in order to preserve fairness in comparison, we use the same SINR formula in maximizing the rate. It is important to note that maximizing the corresponding rate metric, $R=\log (1+\mathrm{SINR})$, is equivalent to the maximization of the SINR metric, since the logarithm is a monotonically increasing function. The maximum SINR optimization problem is formulated as follows,

$$
\begin{aligned}
P_{1}: \max _{\mathbf{u}_{s}} & \text { SINR } \\
\text { s.t. } & \mathfrak{C}_{1}:\left\|\mathbf{u}_{s}\right\|^{2} \leq P_{\max } \\
& \mathfrak{C}_{2}: \operatorname{Pr}_{\mathbf{v}_{p}, \mathbf{H}_{s p}}\left\{\left|\mathbf{v}_{p}^{\dagger} \mathbf{H}_{s p} \mathbf{u}_{s}\right|^{2} \leq P\right\} \geq 1-\delta .
\end{aligned}
$$

After incorporating the sensing information in $(17 \mathrm{c}), \mathfrak{C}_{2}$ is reformulated as follows,

$$
\begin{aligned}
& \operatorname{Pr}_{\mathbf{v}_{p}, \mathbf{H}_{s p}}\left\{\left|\mathbf{v}_{p}^{\dagger} \mathbf{H}_{s p} \mathbf{u}_{s}\right|^{2} \leq P \mid \mathcal{A}\right\} \operatorname{Pr}\{\mathcal{A}\}+ \\
& \operatorname{Pr}_{\mathbf{v}_{p}, \mathbf{H}_{s p}}\left\{\left|\mathbf{v}_{p}^{\dagger} \mathbf{H}_{s p} \mathbf{u}_{s}\right|^{2} \leq P \mid \mathcal{A}^{c}\right\} \operatorname{Pr}\left\{\mathcal{A}^{c}\right\} \geq 1-\delta
\end{aligned}
$$

Considering our sensing scheme we split $P$ depending on the occurrence of $\mathcal{A}$ or $\mathcal{A}^{c}$, as follows,

$$
P=\left\{\begin{array}{ll}
Q_{\text {int }} & ; \mathcal{A} \\
P_{\text {ave }} & ; \mathcal{A}^{c}
\end{array} .\right.
$$

Our objective is to tune the interference threshold $P$ depending on the sensing information. Intuitively, if the primary is active, then $P$ is set to the lowest value, otherwise, $P$ can be arbitrary high. It is then clear that $Q_{\text {int }} \leq P_{\text {ave }}$.

As discussed in section II-A we consider two scenarios which affect $\mathfrak{C}_{2}$ based on our knowledge of the $\mathbf{H}_{s p}$ channel.

\section{A. Scenario 1: where both SR and ST have no knowledge about $\mathbf{H}_{s p}$ and $\mathbf{v}_{p}$}

In this scenario, the constraint $\mathfrak{C}_{2}$ is tackled as follows. We note that $\mathbf{v}_{p}$ and $\mathbf{H}_{s p}$ are independent upon conditioning on $\mathbf{v}_{p}$. Then, the quantity $\mathbf{v}_{p}^{\dagger} \mathbf{H}_{s p} \mathbf{u}_{s}$ is a complex Gaussian random variable with mean 0 and variance $\left\|\mathbf{u}_{s}\right\|^{2}$, recall that $\left\|\mathbf{v}_{p}\right\|^{2}=1$. Note that the conditional distribution of the quantity $\mathbf{v}_{p}^{\dagger} \mathbf{H}_{s p} \mathbf{u}_{s}$ is independent of $\mathbf{v}_{p}$. Therefore, unconditionally $\mathbf{v}_{p}^{\dagger} \mathbf{H}_{s p} \mathbf{u}_{s} \sim \mathcal{C N}\left(0,\left\|\mathbf{u}_{s}\right\|^{2}\right)$. It follows that $\left|\mathbf{v}_{p}^{\dagger} \mathbf{H}_{s p} \mathbf{u}_{s}\right|^{2}$ is exponentially distributed, with parameter $\frac{1}{\left\|\mathbf{u}_{s}\right\|^{2}}$. Therefore, (18) is expressed as,

$$
\begin{aligned}
& {\left[1-\exp \left(-\frac{Q_{\text {int }}}{\left\|\mathbf{u}_{s}\right\|^{2}}\right)\right] \operatorname{Pr}\{\mathcal{A}\}+} \\
& {\left[1-\exp \left(-\frac{P_{\text {ave }}}{\left\|\mathbf{u}_{s}\right\|^{2}}\right)\right] \operatorname{Pr}\left\{\mathcal{A}^{c}\right\} \geq 1-\delta,}
\end{aligned}
$$

Note that (20) is nonlinear with no explicit formula for its zero. Thus, only a numerical solution for the zero of the corresponding equality of (20) can be obtained. Let the left hand side of (20) noted as $F_{p 1}\left(\left\|\mathbf{u}_{s}\right\|^{2}\right)$, then it can be shown, as follows, that $F_{p 1}\left(\left\|\mathbf{u}_{s}\right\|^{2}\right)$ is an invertible function. Differentiating $F_{p 1}\left(\left\|\mathbf{u}_{s}\right\|^{2}\right)$ with respect to $\left\|\mathbf{u}_{s}\right\|^{2}$ results in a negative function. Therefore, $F_{p 1}\left(\left\|\mathbf{u}_{s}\right\|^{2}\right)$ is a monotonically decreasing function, and thus it is invertible function. It follows that (20) can be rewritten as follows,

$$
\left\|\mathbf{u}_{s}\right\|^{2} \leq F_{p 1}^{-1}(1-\delta),
$$

where, $F_{p 1}^{-1}(1-\delta)$ is the inverse function of the left hand side 
of (20). Let $\beta_{1}$ be defined as,

$$
\beta_{1}=\min \left(P_{\max }, F_{p 1}^{-1}(1-\delta)\right) .
$$

Both constraints of problem (17) $\mathfrak{C}_{1}$ and $\mathfrak{C}_{2}$ are reduced to,

$$
\left\|\mathbf{u}_{s}\right\|^{2} \leq \beta_{1}
$$

From (17a), the beamforming receiving vector that maximizes the SINR at the SR, is expressed as $\mathbf{v}_{s}=\mu_{s} \boldsymbol{\Phi}^{-1} \mathbf{H}_{s} \mathbf{u}_{s}$, where, $\mathbf{\Phi}=\left(\mathbf{H}_{p s} \mathbf{x}_{p}\right)\left(\mathbf{H}_{p s} \mathbf{x}_{p}\right)^{\dagger}+N_{0} \mathbf{I}$, and $\mu_{s}=\frac{1}{\left\|\mathbf{\Phi}^{-1} \mathbf{H}_{s} \mathbf{u}_{s}\right\|}$ [30]. Note that to obtain $\boldsymbol{\Phi}$ we do not need to know $\mathbf{H}_{p s}$ or $\mathbf{x}_{p}$, since we are concerned with the interference plus noise term as a whole quantity, and this can be measured frequently when ST stops transmitting. Using the above expressions of $\boldsymbol{\Phi}$ and $\mathbf{v}_{s}$ the SINR in (16) is reformulated as follows,

$$
\mathrm{SINR}=\frac{\mathbf{u}_{s}^{\dagger} \mathbf{H}_{s}^{\dagger} \mathbf{v}_{s} \mathbf{v}_{s}^{\dagger} \mathbf{H}_{s} \mathbf{u}_{s}}{\operatorname{Tr}\left[\mathbf{v}_{s} \mathbf{v}_{s}^{\dagger} \mathbf{H}_{p s} \mathbf{x}_{p} \mathbf{x}_{p}^{\dagger} \mathbf{H}_{p s}^{\dagger}\right]+N_{0}}=\mathbf{u}_{s}^{\dagger} \mathbf{B} \mathbf{u}_{s},
$$

where $\mathbf{B}=\mathbf{H}_{s}^{\dagger} \boldsymbol{\Phi}^{-1} \mathbf{H}_{s}$. It follows that problem (17) becomes,

$$
\begin{array}{ll}
P_{1}^{\prime}: \operatorname{Max} & \mathbf{u}_{s}^{\dagger} \mathbf{B} \mathbf{u}_{s} \\
& \left\|\mathbf{u}_{s}\right\|^{2} \leq \beta_{1}
\end{array}
$$

Problem $P_{1}^{\prime}$ is known as the eigenvalue problem. Its solution can be derived by finding the largest eigenvalue of matrix $\mathbf{B}$ and its associated eigenvector, $\mathbf{b}_{m}$. The solution of $P_{1}^{\prime}$ is expressed exactly as $\sqrt{\beta_{1}} \mathbf{b}_{m}$. Note that there are many methods to numerically find this solution such as, QR, shifted inverse iteration, Rayleigh quotient, and the power iteration methods [31]. It is known that the power iteration method is an algorithm that can quickly compute the largest eigenvalue and its associated eigenvector, provided that the largest eigenvalue is real and distinct. Please note that the convergence rate of this algorithm is decided by the ratio between the second largest eigenvalue and the largest eigenvalue. The smaller the ratio the quicker the convergence rate.

\section{B. Scenario 2: where both SR and ST share perfect knowledge of $\mathbf{H}_{s p}$}

Under scenario 2, (18) is expressed as,

$$
\begin{aligned}
& \operatorname{Pr}_{\mathbf{v}_{p}}\left\{\left|\mathbf{v}_{p}^{\dagger} \mathbf{u}\right|^{2} \leq P_{\text {ave }} \mid \mathcal{A}, \mathbf{H}_{s p}\right\} \operatorname{Pr}\{\mathcal{A}\}+ \\
& \operatorname{Pr}_{\mathbf{v}_{p}}\left\{\left|\mathbf{v}_{p}^{\dagger} \mathbf{u}\right|^{2} \leq Q_{\text {int }} \mid \mathcal{A}^{c}, \mathbf{H}_{s p}\right\} \operatorname{Pr}\left\{\mathcal{A}^{c}\right\} \geq 1-\delta
\end{aligned}
$$

where $\mathbf{u}=\mathbf{H}_{s p} \mathbf{u}_{s}$, is a known vector at ST and SR.

In order to solve (26), we need to obtain the distribution of $\mathbf{v}_{p}$. In [24] Corollary 1, it has been shown that when the PR is deploying either a Matched-Filter (MF), a Zero-Forcing (ZF), or a Minimum-Mean-Squared-Error (MMSE) the distribution of $\mathbf{v}_{p}$ follows a normalized complex Gaussian distribution $\mathcal{C N}(\mathbf{0}, \mathbf{I})$. Similar to the analysis in IV-A, the quantity $\left|\mathbf{v}_{p}^{\dagger} \mathbf{u}\right|^{2}$ follows an exponential distribution, with parameter $\frac{1}{\|\mathbf{u}\|^{2}}$. It follows that (26) is reformulated as,

$$
\begin{aligned}
& {\left[1-\exp \left(-\frac{Q_{\text {int }}}{\|\mathbf{u}\|^{2}}\right)\right] \operatorname{Pr}\{\mathcal{A}\}+} \\
& {\left[1-\exp \left(-\frac{P_{\text {ave }}}{\|\mathbf{u}\|^{2}}\right)\right] \operatorname{Pr}\left\{\mathcal{A}^{c}\right\} \geq 1-\delta,}
\end{aligned}
$$

Similarly to (20), the zero of the corresponding equality of (27) can be obtained numerically. Using a similar derivation method to (21), Inequality (27) can be reformulated as follows,

$$
\|\mathbf{u}\|^{2} \leq F_{p 2}^{-1}(1-\delta)
$$

where $F_{p 2}^{-1}(1-\delta)$ is the inverse function of the left hand side of (27).

To solve problem $P_{1}$ (under scenario 2 assumptions) we introduce new variables, $\mathbf{S}=\mathbf{u}_{s} \mathbf{u}_{s}^{\dagger}, \mathbf{R}=\mathbf{H}_{s p}^{\dagger} \mathbf{H}_{s p}$. Then problem $P_{1}$ becomes,

$$
\begin{aligned}
P_{1}^{\prime \prime}: \quad \max _{\mathbf{S}} & \operatorname{Tr}[\mathbf{B S}] \\
\text { s.t. } & \operatorname{Tr}[\mathbf{S}] \leq P_{\max } \\
& \operatorname{Tr}[\mathbf{R S}] \leq F_{p 2}^{-1}(1-\delta) \\
& \mathbf{S} \succeq 0 \\
& \operatorname{rank}[\mathbf{S}]=1
\end{aligned}
$$

where constraint (29d) is a positive semidefinite condition enforced on variable $\mathbf{S}$, the operator $\operatorname{Tr}[$.$] is the trace of its$ argument matrix, and $\operatorname{Tr}[\mathbf{B S}]=\mathbf{u}_{s}^{\dagger} \mathbf{B} \mathbf{u}_{s}$. Constraint (29e) guarantees that the optimization matrix $\mathbf{S}$ is rank-1 matrix, interpreted as $\mathbf{S}=\mathbf{u}_{s} \mathbf{u}_{s}^{\dagger}$. Note that problem $P_{1}^{\prime \prime}$ is originally a quadratic problem (which is represented as an SDP with rank-1 constraint problem) with only two quadratic constraints, each is represented as an affine constraints of a positive semidefinite matrices. This setting is similar to the one discussed by Huang, Maio, and Zhang in Chapter 6 of [32]. They proved in Theorem 6.4 of [32], that solving an SDP problem with this setting after dropping the rank-1 constraint has the same optimal solution of the original problem without dropping the rank constraint, and it is solved in a polynomial time by an interior-point method, implemented in e.g. CVX Matlab [33],[25].

\section{MAXIMIZING EE}

In this section, we maximize the EE metric subject to minimum rate, peak power, and interference constraints. The received signal at the SR is defined as in (12) or after postcoding as in (13). Utilizing the expression of SINR in (16), the SU rate expression is $R=\log (1+$ SINR $)$, we define the $\mathrm{EE}$ as follows [16], [34],

$$
\mathrm{EE}=\frac{R}{P_{s}}=\frac{\log \left(1+\frac{\left(\mathbf{v}_{s}^{\dagger} \mathbf{H}_{s} \mathbf{u}_{s}\right)^{\dagger}\left(\mathbf{v}_{s}^{\dagger} \mathbf{H}_{s} \mathbf{u}_{s}\right)}{\left(\mathbf{v}_{s}^{\dagger} \mathbf{H}_{p s} \mathbf{x}_{p}\right)^{\dagger}\left(\mathbf{v}_{s}^{\dagger} \mathbf{H}_{p s} \mathbf{x}_{p}\right)+N_{0}}\right)}{\left\|\mathbf{u}_{s}\right\|^{2}}
$$

The EE maximization problem is formulated as follows,

$$
\begin{aligned}
P_{2}: \max _{\mathbf{u}_{s}} & \frac{\log (1+\mathrm{SINR})}{\left\|\mathbf{u}_{s}\right\|^{2}} \\
\text { s.t. } & \left\|\mathbf{u}_{s}\right\|^{2} \leq P_{\max } \\
& \operatorname{Pr}_{\mathbf{v}_{p}, \mathbf{H}_{s p}}\left\{\left|\mathbf{v}_{p}^{\dagger} \mathbf{H}_{s p} \mathbf{u}_{s}\right|^{2} \leq P\right\} \geq 1-\delta \\
& \log (1+\mathrm{SINR}) \geq R_{\text {min }},
\end{aligned}
$$

where the constant $R_{\min }$ is the minimum acceptable rate threshold. The newly introduced constraint (31d) is a minimum rate constraint that aims to secure a certain QoS for the secondary user. 
Note that the objective function of problem $P_{2}$ is a fractional problem, which is not an obvious concave problem. Therefore, we transform this problem into a SDP problem and hence we guarantee the existence of an optimum solution. Hereafter, we use the definition of equivalence between two problems as in [35], (i.e., "Two problems are equivalent if from a solution of one, a solution of the other is readily found, and vice versa").

The first step of the SDP transformation is to introduce a new optimization variable $t$ that changes the objective function (31a) into a constraint, $\frac{\log (1+\mathrm{SINR})}{\operatorname{Tr}[\mathbf{S}]} \geq \frac{1}{t}$, while minimizing a different objective function, i.e. $t$, [35]. Furthermore, we enforce a new equality constraint as SINR $=\mathbf{u}_{s} \mathbf{B} \mathbf{u}_{s}^{\dagger}=$ $\operatorname{Tr}[\mathbf{B S}]=\gamma_{s}$, where $\mathbf{S}=\mathbf{u}_{s} \mathbf{u}_{s}^{\dagger}$. Then, problem $P_{2}$ becomes equivalent to the following,

$$
\begin{array}{rl}
P_{2}^{\prime}: \min _{\mathbf{S}, t} & t \\
\text { s.t } & \frac{\log \left(1+\gamma_{s}\right)}{\operatorname{Tr}[\mathbf{S}]} \geq \frac{1}{t} \\
& \operatorname{Tr}[\mathbf{B S}]=\gamma_{s} \\
& \operatorname{Tr}[\mathbf{S}] \leq P_{\max } \\
& \operatorname{Tr}\left[\mathbf{R}_{i} \mathbf{S}\right] \leq F_{p i}^{-1}(1-\delta) \quad \forall i=1,2 \\
& \operatorname{Tr}[\mathbf{B S}]-\left(\exp \left(R_{\min }\right)-1\right) \geq 0 \\
& \mathbf{S} \succeq 0 \\
& \operatorname{rank}[\mathbf{S}]=1,
\end{array}
$$

where, in constraint (32e), $i=1$ corresponds to scenario $1\left(\mathbf{R}_{1}=\mathbf{I}\right)$ and $i=2$ corresponds to scenario $2\left(\mathbf{R}_{2}=\right.$ $\mathbf{H}_{s p}^{\dagger} \mathbf{H}_{s p}$ ). Note that constraints (31b), (31c), and (31d) are equivalent to constraints (32d), (32e), and (32f), respectively. Constraint (32h) is to guarantee that the optimization matrix $\mathbf{S}$ can be written as an outer product of a vector, i.e. $\mathbf{S}=\mathbf{u}_{s} \mathbf{u}_{s}^{\dagger}$, which is interpreted as $\mathbf{S}$ is a rank-1 matrix. Constraint (32c) enforces the newly introduced parameter in $(32 \mathrm{~b}), \gamma_{s}$, to be equivalent to the SINR, thus $\frac{\log \left(1+\gamma_{s}\right)}{\operatorname{Tr}[\mathbf{S}]}=\frac{\log (1+\operatorname{SINR})}{\operatorname{Tr}[\mathbf{S}]}$. This constraint results in transforming constraint (32b) into an affine function of the optimization variables. The equivalence between $P_{2}$ and $P_{2}^{\prime}$ is proved in two steps, one is showing that the optimal solution of $P_{2}$ is optimal for $P_{2}^{\prime}$ while the other is showing that the optimal solution of $P_{2}^{\prime}$ is optimal for $P_{2}$. First, let $\mathbf{u}_{s}^{*}$ be the optimal solution of $P_{2}$ that maximizes $\mathrm{EE}=\frac{\log (1+\mathrm{SINR})}{\left\|\mathbf{u}_{s}\right\|^{2}}$ under (31b), (31c), and (31d). The corresponding $\mathbf{S}^{*}=\mathbf{u}_{s}^{*} \mathbf{u}_{s}^{* \dagger}$ and SINR $^{*}=\operatorname{Tr}\left[\mathbf{B S}^{*}\right]$ achieve similar $\mathrm{EE}=\frac{\log \left(1+\mathrm{SINR}^{*}\right)}{\operatorname{Tr}\left[\mathbf{S}^{*}\right]}$ value under equivalence constraints while minimizing $t$, without violating (32b), up to $t^{*}=\frac{\operatorname{Tr}\left[\mathbf{S}^{*}\right]}{\log \left(1+\text { SINR }^{*}\right)}$. Note that the pair $\left(\mathbf{S}^{*}, t^{*}\right)$ is feasible solution of $P_{2}^{\prime}$. Let us assume that we can achieve an optimal solution for $P_{2}^{\prime}$ that is better than the pair $\left(\mathbf{S}^{*}, t^{*}\right)$. Then it follows that this new optimal solution either violates one of the $P_{2}^{\prime}$ constraints, or it achieves larger EE than the corresponding $\mathbf{u}_{s}^{*}$ which means that $\mathbf{u}_{s}^{*}$ does not maximize the EE metric, this contradicts with our assumption. Second, assume that the optimal pair of variables $(\mathbf{S}, t)$ that optimizes problem $P_{2}^{\prime}$ is designated as $\left(\mathbf{S}_{2}^{*}\right.$ ,$\left.t_{2}^{*}\right)$. The corresponding optimal EE and SINR values are designated as $\mathrm{EE}_{2}^{*}$ and $\mathrm{SINR}_{2}^{*}$, respectively, and the corresponding vector $\mathbf{u}_{s}$ is designated as $\mathbf{u}_{2}^{*}$. Note that minimizing $\mathrm{t}$, the objective function of $P_{2}^{\prime}$, increases $\frac{1}{t}$ and its corresponding upper bound $\frac{\log (1+\mathrm{SINR})}{\operatorname{Tr}[\mathbf{S}]}$, in constraint $(32 \mathrm{~b})$, up to a certain value such that, $\mathrm{EE}_{2}^{*}=\frac{\log \left(1+\mathrm{SINR}_{2}^{*}\right)}{\operatorname{Tr}\left[\mathbf{S}_{2}^{*}\right]}=\frac{\log \left(1+\mathrm{SINR}_{2}^{*}\right)}{\left\|\mathbf{u}_{2}^{*}\right\|^{2}}$. Note that $\mathbf{u}_{2}^{*}$ satisfies all the constraints (32d), (32e), and (32f), and they do not depend on $t$. Since $\mathbf{u}_{2}^{*}$ maximizes EE under all constraints of $P_{2}^{\prime}$, thus all constraints of $P_{2}$, then $\mathbf{u}_{2}^{*}$ maximizes the EE metric in $P_{2}$ under the corresponding constraints. Now, assume an optimal solution for $P_{2}$ that is better than $\mathbf{u}_{2}^{*}$. This solution either violates $P_{2}$ 's constraints (31b), (31c), and (31d) or the optimal pair $\left(\mathbf{S}_{2}^{*}, t_{2}^{*}\right)$ does not maximize EE, which contradicts with the above assumption. From the first and the second steps it is concluded that $P_{2}$ is equivalent to $P_{2}^{\prime}$.

Problem $P_{2}^{\prime}$, without considering constraint (32h), is an SDP problem but not in the standard form. Thus we introduce dummy variables for the inequality constraints (32b), (32d), (32e), and (32f), under both scenarios, which change them into equality constraints as below.

\section{A. Scenario 1:}

In this scenario, both SR and ST have no knowledge about $\mathbf{H}_{s p}$, both (32d) and (32e) can be combined as we did in IV-A i.e. $\operatorname{Tr}[S] \leq \beta_{1}$. Using the fact that $\operatorname{Tr}[\mathbf{A B}]=$ $\operatorname{vec}\left(\mathbf{A}^{T}\right)^{T} \operatorname{vec}(\mathbf{B})$, where $\operatorname{vec}($.$) is the vectorization operator$ [36] which converts the matrix into a column vector by stacking the columns of the matrix on top of one another, $P_{2}^{\prime}$ can be written as,

$$
\begin{aligned}
\min _{2 a}: & t \\
\text { s.t } & t \log \left(1+\gamma_{s}\right)-\operatorname{vec}(\mathbf{I})^{T} \operatorname{vec}(\mathbf{S})-r_{1}=0 \\
& \operatorname{vec}\left(\mathbf{B}^{T}\right)^{T} \mathbf{v e c}(\mathbf{S})=\gamma_{s} \\
& \beta_{1}-\mathbf{v e c}(\mathbf{I})^{T} \mathbf{v e c}(\mathbf{S})-r_{2}=0 \\
& \operatorname{vec}\left(\mathbf{B}^{T}\right)^{T} \mathbf{v e c}(\mathbf{S})-\left(\exp \left(R_{\text {min }}\right)-1\right)-r_{3}=0 \\
& \mathbf{S} \succeq 0 \\
& r_{i} \geq 0 \quad i=1,2,3 \\
& \operatorname{rank}[\mathbf{S}]=1,
\end{aligned}
$$

The main difference between problem $P_{2}^{\prime}$ and $P_{2 a}$ is the introduced three slackness variables $r_{1}, r_{2}$, and $r_{3}$ to the inequality constraints (33c), (33e), and (33f), respectively. Problem $P_{2 a}$, without the rank-1 constraint, is already a SDP problem, however, we do some manipulation to reformulate into a standard SDP as follows. By defining a new optimization variable $\mathbf{x}=\left[\operatorname{vec}(\mathbf{S})^{T}, t, r_{1}, r_{2}, r_{3}\right]^{T}$, a new matrix

$$
\mathbf{A}=\left[\begin{array}{ccccc}
\operatorname{vec}\left(\mathbf{B}^{T}\right)^{T} & 0 & 0 & 0 & 0 \\
-\operatorname{vec}(\mathbf{I})^{T} & \log \left(1+\gamma_{s}\right) & -1 & 0 & 0 \\
-\operatorname{vec}(\mathbf{I})^{T} & 0 & 0 & -1 & 0 \\
\operatorname{vec}\left(\mathbf{B}^{T}\right)^{T} & 0 & 0 & 0 & -1
\end{array}\right]
$$

and new vectors $\mathbf{b}=\left[\gamma_{s}, 0,-\beta_{1}, e^{R_{\min }}-1\right]^{T}$ and $\mathbf{c}=$ $\left[\mathbf{0}_{N_{t}^{2} X 1}^{T}, 1,0,0,0\right]^{T}$, it is possible to rewrite $P_{2 a}$ (33), after dropping the rank-1 constraint, into standard SDP form as 
follows,

$$
\begin{aligned}
P_{2 a}^{\prime}: \min _{\mathbf{x}} & \mathbf{c}^{T} \mathbf{x} \\
\text { s.t. } & A \mathbf{x}=\mathbf{b} \\
& {[\mathbf{x}]_{k} \geq 0, k=N_{t}^{2}+1, \ldots, N_{t}^{2}+4 . } \\
& \operatorname{mat}\left([\mathbf{x}]_{N_{t}^{2}}^{1}\right)_{N_{t}} \succeq 0
\end{aligned}
$$

where, the operator $[\mathbf{x}]_{k}$ represents the $k^{t h}$ element of vector $\mathbf{x}$, the operator $[\mathbf{x}]_{N_{t}^{2}}^{1}$ represents the elements from index 1 to index $N_{t}^{2}$ of vector $\mathbf{x}$. The term $[\mathbf{x}]_{k} \geq 0$ is an element wise inequality, and the function mat $\left([\mathbf{x}]_{N_{t}^{2}}^{1}\right)_{N_{t}}$ transforms the vector $\mathbf{x}$ to matrix by aligning the first $N_{t}$ elements of $\mathbf{x}$ as the first column in the matrix, and so on. The above problem is recognized as an SDP problem that can be solved by the interior-point method in a polynomial time. It is observed that the rank-1 constraint is dropped in $P_{2 a}^{\prime}$, which means that problem $P_{2 a}^{\prime}$ is a relaxed version of $P_{2 a}$. There are several ways to find the optimal solution of $P_{2 a}$ from the optimal solution of $P_{2 a}^{\prime}$, as follows. One way of finding the optimal solution of the original problem $P_{2 a}$ is to obtain the optimal solution of $P_{2 a}^{\prime}, \mathrm{x}^{*}$, and check the rank-1 constraint as follows, rank $\left[\operatorname{mat}\left(\left[\mathbf{x}^{*}\right]_{N_{t}^{2}}^{1}\right)_{N_{t}}\right]=1$. In case the constraint is satisfied then $\mathrm{x}^{*}$ is optimal for both $P_{2 a}$ and $P_{2 a}^{\prime}$ otherwise we declare an outage event. Note that the complexity of finding the optimal solution in this way consists of two parts. The first, is solving the SDP problem, which is done in polynomial time. The second, is checking the matrix rank, which can be done in many methods, such as, rank-revealing QR factorization method, or singular value decomposition method. Another way of finding the optimal solution of problem $P_{2 a}$, without checking the rank, is to use some randomization methods explained by Luo and Chang in Chapter 4 of [32].

\section{B. Scenario 2}

In this scenario, both SR and ST share perfect knowledge about $\mathbf{H}_{s p}$. Similar to $\mathrm{V}-\mathrm{A}$ we use the fact that $\operatorname{Tr}[\mathbf{A B}]=$ $\operatorname{vec}\left(\mathbf{A}^{T}\right)^{T} \operatorname{vec}(\mathbf{B})$, then $P_{2}^{\prime}$ becomes,

$$
\begin{array}{rl}
P_{2 b}: & \\
\mathbf{S i n}, t, r_{1}, r_{2}, r_{3}, r_{4} & t \\
\text { s.t } & t \log \left(1+\gamma_{s}\right)-\mathbf{v e c}(\mathbf{I})^{T} \mathbf{v e c}(\mathbf{S})-r_{1}=0 \\
& \operatorname{vec}\left(\mathbf{B}^{T}\right)^{T} \mathbf{v e c}(\mathbf{S})=\gamma_{s} \\
& P_{\max }-\mathbf{v e c}(\mathbf{I})^{T} \mathbf{v e c}(\mathbf{S})-r_{2}=0 \\
& F_{p 2}^{-1}(1-\delta)-\mathbf{v e c}(\mathbf{R})^{T} \mathbf{v e c}(\mathbf{S})-r_{3}=0 \\
& \operatorname{vec}\left(\mathbf{B}^{T}\right)^{T} \mathbf{v e c}(\mathbf{S})-\left(\exp \left(R_{\min }\right)-1\right)-r_{4}=0 \\
& \\
& \mathbf{S} \succeq 0 \\
& r_{i} \geq 0 \\
& \operatorname{rank}[\mathbf{S}]=1,
\end{array}
$$

Note that (36e) and (36f) are not combined into one constraint, and they are similar to the power constraint in (29b) and interference constraint in (29c), and $r_{1}, r_{2}, r_{3}$, and $r_{4}$ are the corresponding slackness variables to the inequality constraints (36c), (36e), (36f), and (36g), respectively. Similarly to V-A we can rewrite problem (36) into a standard SDP problem, without rank constraint, and solve it in a similar way, while taking into consideration that constraints (36e) and (36f) are different than (33e).

\section{Numerical RESULtS}

In this section, we evaluate the proposed schemes with sensing information versus the same schemes without sensing information which are considered as benchmarks. We consider the parameters mentioned in Table I to evaluate the schemes. The evaluation is done by checking the SU maximum achievable EE metric (in bits/Joule/Hz), the SU rate (in bits/sec/Hz) that corresponds to the maximum SINR, and the outage metric, which is defined as the event where there is no feasible solution of the corresponding optimization problem.

TABLE I SIMULATION PARAMETERS

\begin{tabular}{|l|l|}
\hline Parameter Name & Value \\
\hline Sensing averaging bits & 1000 \\
\hline$P_{\max }$ and $P_{\text {ave }}$ & $27 \mathrm{dBm}[37]$ \\
\hline Maximum PT power $\left(P_{p}\right)$ & $27 \mathrm{dBm}$ \\
\hline \# Monte-Carlo iterations & 10000 \\
\hline Wireless channels & $\begin{array}{l}\text { Rayleigh, } \\
\text { Slow Flat Fading }\end{array}$ \\
\hline$\left(N_{t} ; N_{r}\right)$ & $(4 ; 4)$ \\
\hline$\left(M_{t} ; M_{r}\right)$ & $(2 ; 2)$ \\
\hline$Q_{\text {int }}$ & $0 \mathrm{dBm}, 3 \mathrm{dBm}$ \\
\hline Minimum Rate $\left(R_{\min }\right)$ & $0.1 \mathrm{bits} / \mathrm{sec} / \mathrm{Hz}$ \\
\hline$\delta$ & 0.1 \\
\hline$\alpha$ & $0.15,0.2$ \\
\hline$P_{D}, P_{F A}$ & $0.9,0.01$ \\
\hline
\end{tabular}

We begin by discussing the results of maximizing the EE metric. Knowing that the maximum EE is resulted from minimizing the power and the corresponding rate. However, note that we have a minimum rate constraint. Therefore, we define the EE outage probability metric, which is the probability of outage that happens because there is no feasible solution that satisfies all the problem's constraints, as follows,

$$
\text { EE Outage }= \begin{cases}0 & ; \text { A feasible solution exists } \\ 1 & ; \text { No feasible solution }\end{cases}
$$

Figure 2, shows the performance of all schemes with and without sensing information versus $Q_{i n t}$. The performance of "with sensing, scenario 2" scheme achieves the lowest outage probability around 0.12 at $Q_{\text {int }}=-10 \mathrm{dBm}$, which outperforms the performance of "without sensing, scenario 2" scheme that achieves 0.28 at $Q_{\text {int }}=-10 \mathrm{dBm}$. On the other hand, the margin of outage probability improvement achieved by "With sensing, scenario 1" scheme comparing to "no sensing, scenario 1" scheme is about 0.9 around $Q_{\text {int }}=-4 \mathrm{dBm}$. It is observed that by increasing $Q_{\text {int }}$ the outage probability decreases for all schemes. Note that the schemes which utilizes sensing outperform the ones without sensing by around $7 \mathrm{dBm}$ for scenario 2 and $9 \mathrm{dBm}$ for scenario 1 at outage probability $\approx 0.1$. 


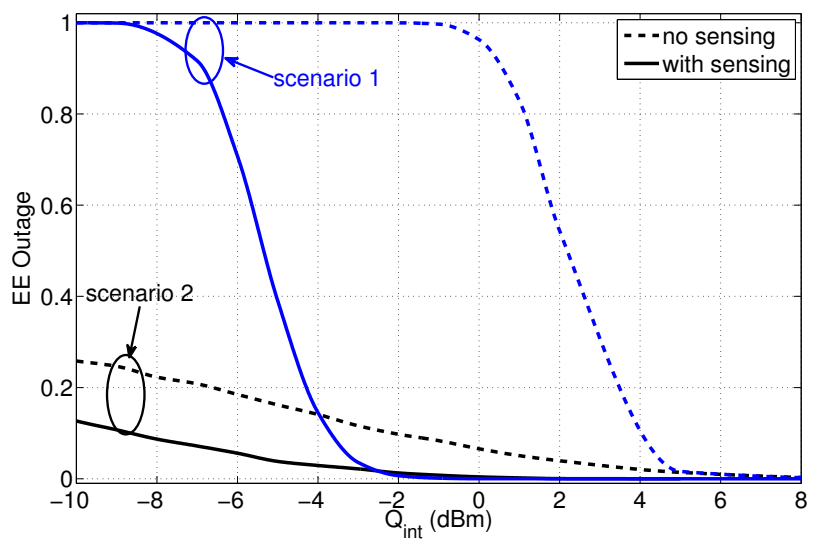

Fig. 2. EE outage probability for all schemes versus $Q_{\text {int }}$.

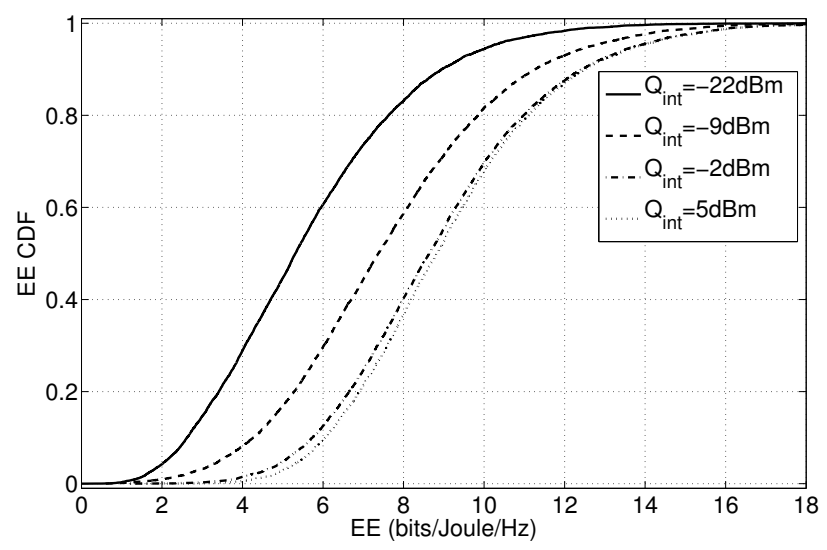

Fig. 3. EE CDF of scenario 2 with sensing scheme for several $Q_{\text {int }}$.

Figure 3, shows the CDF of "with sensing, scenario 2" scheme for different values of $Q_{\text {int }}$. It is observed that increasing the values of $Q_{i n t}$ results in improving the EE performance of the system. Furthermore, increasing $Q_{i n t}$ more than a certain value does not improve the performance.

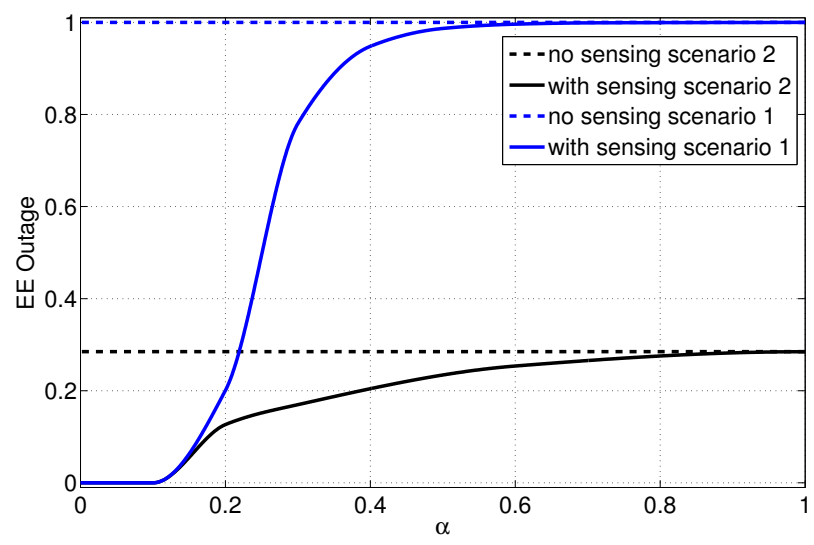

Fig. 4. EE Outage Versus $\alpha$ for all schemes.

Figure 4, shows the EE outage probability performance of the proposed schemes versus the parameter $\alpha$, where $\alpha$ follows a Bernoulli distribution and it expresses the prior probability of PU existence $\alpha=\operatorname{Pr}\left\{\mathcal{H}_{1}\right\}$. As described earlier, $\operatorname{Pr}\{\mathcal{A}\}$ is a function of $\alpha$ and the sensor characteristics $P_{D}$ and $P_{F A}$. Furthermore, the value of $\operatorname{Pr}\{\mathcal{A}\}$ is affected by the change in $\alpha$. Figure 4 shows that the "with sensing, scenario 2" scheme outperforms the "with sensing, scenario 1", which is expected since "with sensing, scenario 2" utilizes full knowledge of the ST-PR channel. It is important to note that as $\alpha \rightarrow 1$ the performance of "with sensing, scenario 2" scheme degrades untill it converges to the performance of "no sensing, scenario 2" scheme. A similar observation can be noted for "with sensing, scenario 1" scheme which converges to "no sensing, scenario 1" scheme as $\alpha \rightarrow 1$.

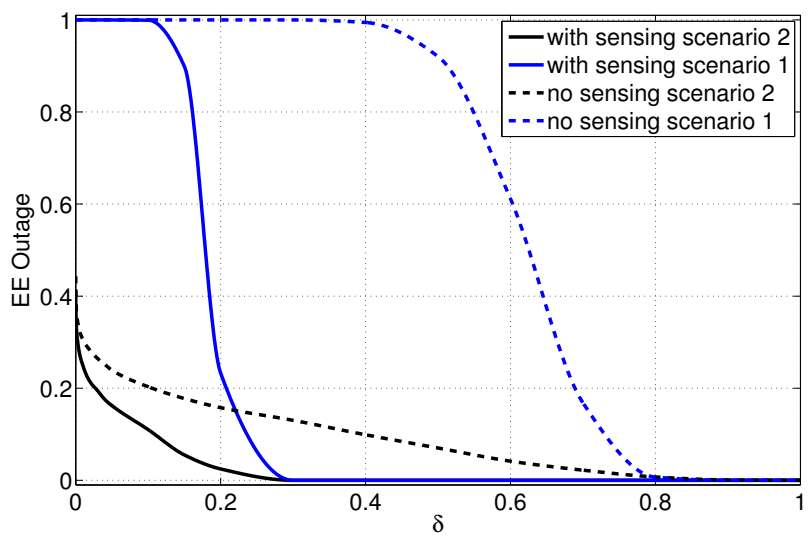

Fig. 5. EE outage versus $\delta$ for all schemes.

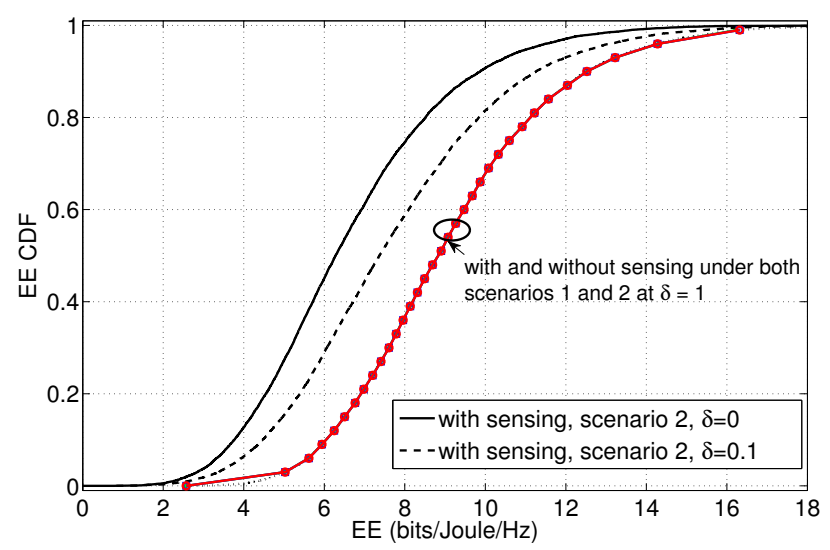

Fig. 6. EE CDF Versus $\delta$ for all schemes.

The outage performance of the all schemes versus $\delta$ is shown in Fig. 5. Note that the interpretation of $\delta$ reflects on the secondary to primary interference constraint. The higher the value of $\delta$ the more relaxed the interference constraints. The lower the value of $\delta$ the stricter the interference constraints. From Fig. 5 we note that by increasing $\delta$ up to 1 all schemes converges to a zero outage probability. Which means that at $\delta=1$ the interference constraint has no effect on the problem at all. It is observed that the "with sensing, scenario 2" scheme dominate the outage performance of all schemes. Again, it is 
clear that the with sensing schemes outperform the without sensing schemes with outage difference varies from $\approx 0$ up to $\approx 1$.

Figure 6 shows the EE CDF performance of the proposed scheme scenario 2 for several values of $\delta=0, \delta=0.1$, and $\delta=1$. It is observed that the "with sensing, scenario 2 " scheme improved rapidly by increasing the values of $\delta$. It is important to note that at $\delta=1$ all proposed and benchmark schemes reach similar CDF performance, which is consistent with the EE outage performance in Fig. 5.

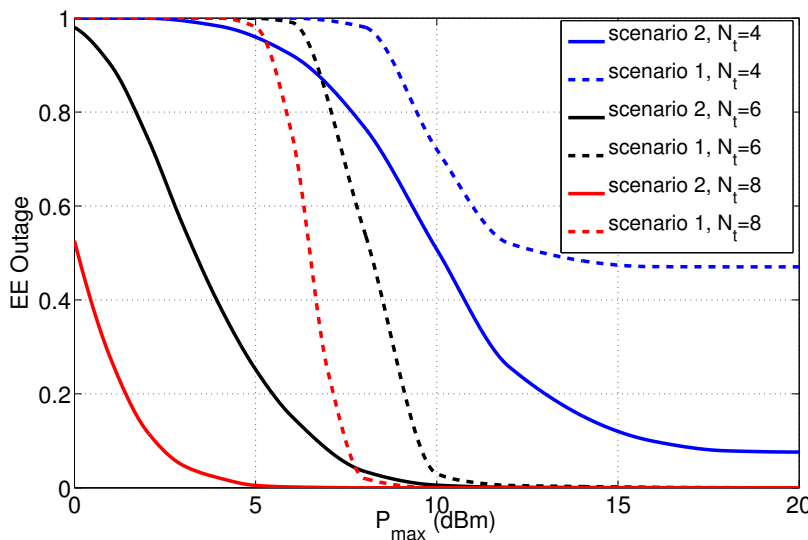

Fig. 7. EE outage versus $P_{\max }$ and different $N_{t}=4,6,8$ and for all with sensing schemes.

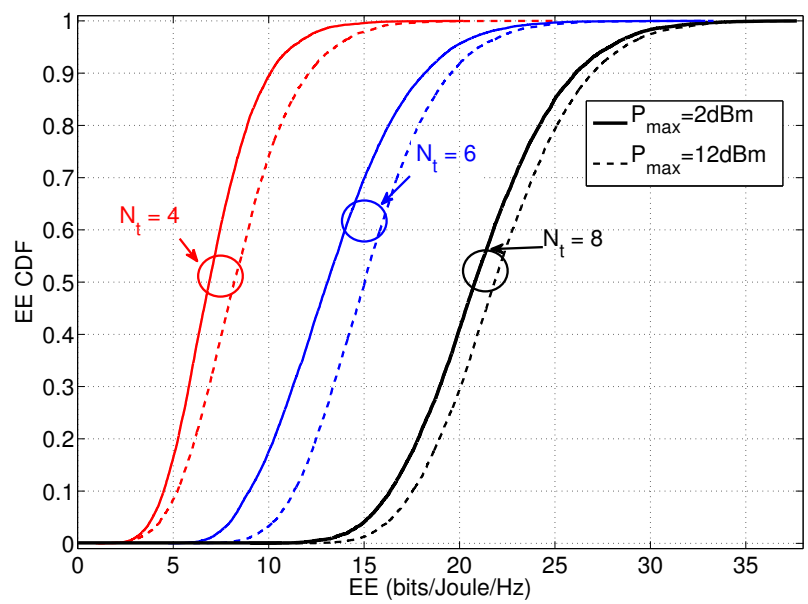

Fig. 8. EE CDF versus for different values of $N_{t}$ and $P_{\max }$ for "with sensing, scenario 2" scheme.

We show the outage probability of the with sensing schemes for both "scenario 2" and "scenario 1" versus $P_{\max }$ and different number of antennas, $N_{t}$, in Fig. 7. We note the effect of decreasing the $P_{\max }$ on degrading the performances of both schemes for different values of $N_{t}$, namely, $N_{t}=4, N_{t}=6$, and $N_{t}=8$. We observe the EE outage improvement by increasing $N_{t}$. The improvement of scenario 2 comparing to scenario 1 is observed for different values of $N_{t}$. Furthermore, Fig. 8 shows the EE CDF improvement for scenario 2 with sensing information by increasing $N_{t}$ and $P_{\max }$.
In the next part of this section we discuss the numerical result of maximizing the SINR versus different parameters. The evaluation metric of the SINR is the CDF of the corresponding rate.

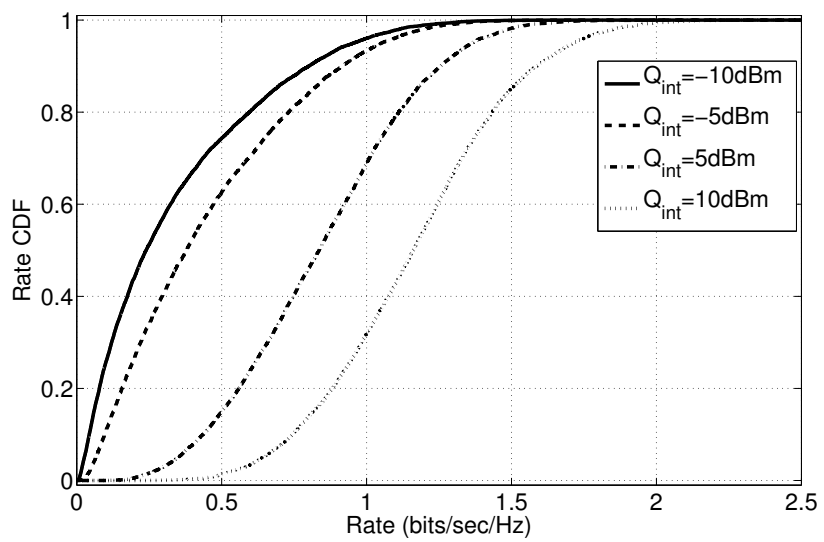

Fig. 9. $\mathrm{CDF}$ of the Rate with variable $Q_{\text {int }}$ for with sensing, scenario 2 scheme.

Figure 9 shows the performance of the proposed schemes with sensing information under scenario 2 versus $Q_{i n t}$. It is observed that the rate performance is improved by increasing the value of $Q_{\text {int }}$.

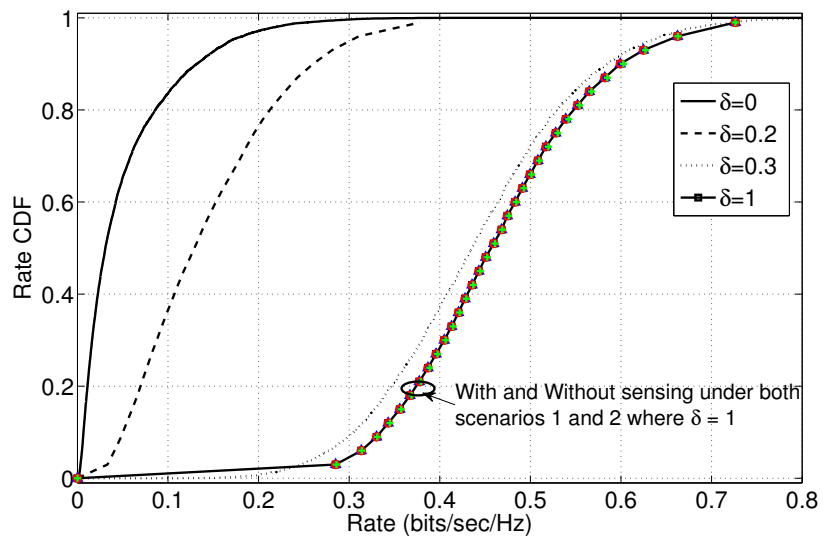

Fig. 10. CDF of the Rate versus $\delta$ for all schemes.

The performance of the proposed schemes versus $\delta$ is shown in Fig. 10. Note as explained above, that the interpretation of $\delta$ reflects on the secondary to primary interference constraint. The higher the value of $\delta$ the more relaxed the interference constraints is. Hence, we expect an improved Rate by increasing $\delta$. On the other hand, the lower the value of $\delta$ the stricter is the interference constraints. It is observed from Fig. 10 that the CDF performance for all the schemes with and without sensing for both scenarios have exactly the same performance at $\delta=1$. We note that the CDF performance degrades by decreasing the value of $\delta$ for both with and without sensing scenario-2, where $\delta=1,0.3,0.2$ and 0 .

Figure 11, shows the performance of the proposed and benchmark schemes versus $\alpha$. To emphasize on what described 


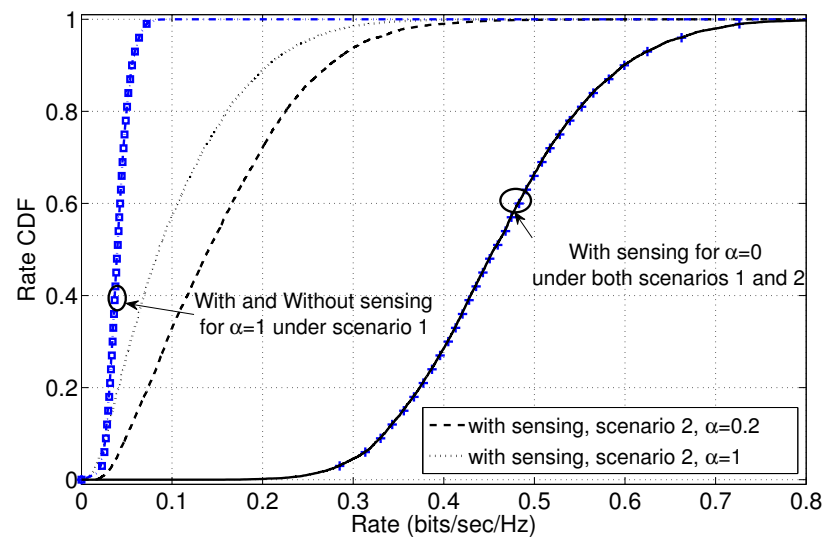

Fig. 11. $\mathrm{CDF}$ of the Rate for all schemes with variable $\alpha$.

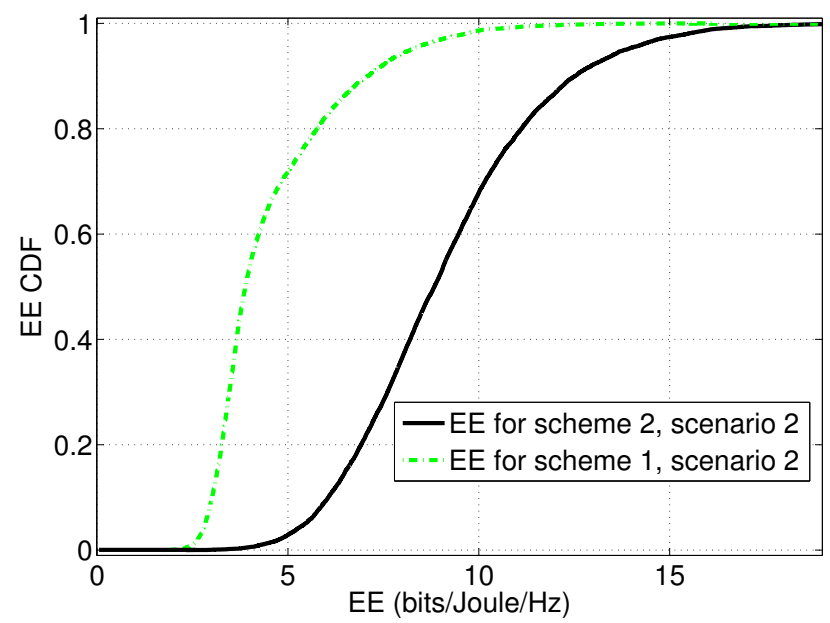

Fig. 12. EE performance comparison between scheme 1 (maximum SINR) and scheme 2 (maximum EE).

earlier, we know that $\alpha$ is a function of $\operatorname{Pr}\{\mathcal{A}\}$, hence, any change in $\alpha$ results in a change in $\operatorname{Pr}\{\mathcal{A}\}$. It is observed from Fig. 11 that for $\alpha=1$ both schemes (with and without sensing) of scenario 1 have exactly the same performance, note that the schemes which do not use the sensing information is not affected by changing $\alpha$. This result is realistic, since at $\alpha=1$ we need to protect the primary at all times, hence the sensing information is not useful. We also note that at $\alpha=0$ the schemes that utilize the sensing information under both scenarios 1 and 2 have similar performance. This is a realistic result since whether the ST have knowledge on the ST-PR channel or not, it is always transmitting because the PU is idle. We also note that by increasing $\alpha$ the performance of the both proposed schemes degrades, which is depicted for $\alpha=0$, 0.2 , and 1 , under scenario 2 .

Figure 12 shows an energy efficiency performance comparison between both scheme 1 (maximizing SINR metric) and scheme 2 (maximizing EE metric). It is observed that scheme 2 achieves better energy efficiency performance than scheme 1. Which is expected since scheme 2 maximizes the EE metric, whereas, scheme 1 maximizes the SINR metric. This shows

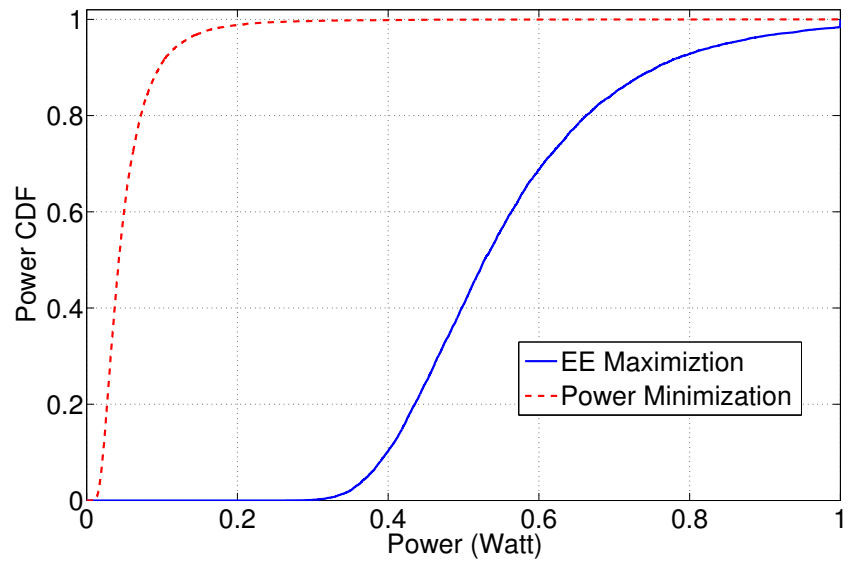

Fig. 13. Comparison of the power CDF performance between the energy efficiency maximization problem and the power minimization problem for $N_{t}=N_{r}=2$ and $M_{t}=M_{r}=2$.

that both of the maximization problems are not equivalent as claimed earlier.

Figure 13 shows the CDF of the power for both energy efficiency maximization and power minimization problems under $N_{t}=N_{r}=2, M_{t}=M_{r}=2$, and a minimum rate threshold $R_{\text {min }}=0.1 \mathrm{bits} / \mathrm{sec} / \mathrm{Hz}$. As expected, it is observed that the optimal power needed for the power minimization scheme is less than that of the energy efficiency maximization scheme with probability $=1$. It is noted that for a certain probability of power $\leq 0.9$, the optimal required power of the power minimization scheme is about 0.1 Watts, whereas, the corresponding power of the EE maximization scheme is about 0.75 Watts.

\section{CONCLUSION}

This paper proposed two energy efficient beamforming schemes based on cognitive radio environments. The proposed beamforming schemes utilize the sensing information obtained from the secondary user multi-antenna sensor using a blind combining technique integrated with an energy detector. Furthermore, we investigated the effect of the knowledge of the secondary to primary channel through two scenarios, full knowledge and no knowledge. Some of the optimization problem of the proposed schemes are not a convex problem. Therefore, we convert them to a SDP standard problem to guarantee the global unique solution. We evaluated and analyzed the proposed schemes. The numerical results show a tremendous energy efficiency, rate, and outage performance improvement for both scenarios with sensing information compared to the ones without sensing. Furthermore, selected numerical result shows a considerable energy efficiency gain of scheme 2 compared to scheme 1 and the power minimization problems.

\section{REFERENCES}

[1] A. Anand, C. Manikopoulos, Q. Jones, and C. Borcea, "A quantitative analysis of power consumption for location-aware applications on smart phones," in IEEE International Symposium on Industrial Electronics. ISIE 2007, 2007, pp. 1986-1991. 
[2] C. Han, T. Harrold, and et al., "Green radio: radio techniques to enable energy-efficient wireless networks," Communications Magazine, IEEE, vol. 49, no. 6, pp. $46-54$, june 2011.

[3] Z. Hasan, H. Boostanimehr, and V. Bhargava, "Green cellular networks: A survey, some research issues and challenges," Communications Surveys Tutorials, IEEE, vol. 13, no. 4, pp. $524-540$, quarter 2011.

[4] D. Feng, C. Jiang, G. Lim, J. Cimini, L.J., G. Feng, and G. Li, "A survey of energy-efficient wireless communications," IEEE Communications Surveys Tutorials, vol. 15, no. 1, pp. 167-178, 2013.

[5] G. Li, Z. Xu, C. Xiong, C. Yang, S. Zhang, Y. Chen, and S. Xu, "Energyefficient wireless communications: tutorial, survey, and open issues," IEEE Wireless Communications, vol. 18, no. 6, pp. 28-35, 2011.

[6] A. Alabbasi, Z. Rezki, and B. Shihada, "Energy efficiency and SINR maximization beamformers for cognitive radio utilizing sensing information," in IEEE International Symposium on Information Theory (ISIT), 2014, accepted.

[7] Q. Zhao and B. Sadler, "A survey of dynamic spectrum access," Signal Processing Magazine, IEEE, vol. 24, no. 3, pp. 79-89, 2007.

[8] C. Shen and M. Fitz, "Dynamic spatial spectrum access with opportunistic orthogonalization," in 43rd Annual Conference on Information Sciences and Systems, 2009., 2009, pp. 600-605.

[9] S. Srinivasa and S. Jafar, "Soft sensing and optimal power control for cognitive radio," IEEE Transactions on Wireless Communications, vol. 9, no. 12 , pp. $3638-3649$, december 2010.

[10] R. Fischer and J. Huber, "A new loading algorithm for discrete multitone transmission," in Global Telecommunications Conference, 1996. GLOBECOM '96. 'Communications: The Key to Global Prosperity, vol. 1, nov 1996, pp. $724-728$ vol.1.

[11] J. Solares, Z. Rezki, and M.-S. Alouini, "Optimal power allocation of a sensor node under different rate constraints," in IEEE International Conference on Communications (ICC), 2012, June 2012, pp. 2334-2338.

[12] R. Zhang, S. Cui, and Y.-C. Liang, "On ergodic sum capacity of fading cognitive multiple-access and broadcast channels," IEEE Transactions on Information Theory, vol. 55, no. 11, pp. 5161-5178, Nov 2009.

[13] R. S. Prabhu and B. Daneshrad, "An energy-efficient water-filling algorithm for OFDM systems," in IEEE International Conference on Communications (ICC), 2010, 2010, pp. 1-5.

[14] A. Alabbasi, Z. Rezki, and B. Shihada, "Energy efficient scheme for cognitive radios utilizing soft sensing," in IEEE Wireless Communications and Networking Conference (WCNC), 2014, 2014, pp. 1-6.

[15] R. Prabhu, Performance Analysis of Energy-efficient Adaptive Modulation, 2010. [Online]. Available: http://books.google.com.sa/ books?id=QvbeZwEACAAJ

[16] C. Jiang and L. Cimini, "Energy-efficient multiuser MIMO beamforming," in 45th Annual Conference on Information Sciences and Systems (CISS), 2011, pp. 1-5.

[17] D. Gesbert, M. Shafi, D. shan Shiu, P. Smith, and A. Naguib, "From theory to practice: an overview of MIMO space-time coded wireless systems," IEEE Journal on Selected Areas in Communications, vol. 21 , no. 3, pp. 281-302, 2003.

[18] R. Blum, "MIMO capacity with interference," IEEE Journal on Selected Areas in Communications, vol. 21, no. 5, pp. 793-801, 2003.

[19] K. Cumanan, R. Zhang, and S. Lambotharan, "A new design paradigm for mimo cognitive radio with primary user rate constraint," IEEE Communications Letters, vol. 16, no. 5, pp. 706-709, May 2012.

[20] S.-J. Kim and G. Giannakis, "Optimal resource allocation for MIMO ad hoc cognitive radio networks," IEEE Transactions on Information Theory, vol. 57, no. 5, pp. 3117-3131, 2011.

[21] R. Zhang and Y.-C. Liang, "Exploiting multi-antennas for opportunistic spectrum sharing in cognitive radio networks," IEEE Journal of Selected Topics in Signal Processing, vol. 2, no. 1, pp. 88-102, 2008.

[22] H. Volos and R. Buehrer, "Cognitive engine design for link adaptation: An application to multi-antenna systems," IEEE Transactions on Wireless Communications, vol. 9, no. 9, pp. 2902 -2913, september 2010.

[23] B. Fette, Cognitive Radio Technology.

[24] Y. Zhang and A. So, "Optimal spectrum sharing in MIMO cognitive radio networks via semidefinite programming," IEEE Journal on Selected Areas in Communications, vol. 29, no. 12, pp. 362 - 373, February 2011.

[25] A. Ben-Tal and A. S. Nemirovskiaei, Lectures on Modern Convex Optimization: Analysis, Algorithms, and Engineering Applications. Philadelphia, PA, USA: Society for Industrial and Applied Mathematics, 2001.

[26] A. Al-Abbasi and T. Fujii, "A novel blind diversity detection scheme for multi-antenna cognitive radio spectrum sensing," in Vehicular Technology Conference Fall (VTC 2010-Fall), IEEE 72nd, 2010, pp. 1-5.
[27] M. Kang and M.-S. Alouini, "Largest eigenvalue of complex Wishart matrices and performance analysis of MIMO mrc systems," IEEE Journal on Selected Areas in Communications, vol. 21, no. 3, pp. 418426, 2003.

[28] I. S. Gradshteyn and I. M. Ryzhik, Table of Integrals, Series, and Products, Seventh Edition, 7th ed. Academic Press, Jan.

[29] L. Zhang, Y.-C. Liang, and Y. Xin, "Joint beamforming and power allocation for multiple access channels in cognitive radio networks," IEEE Journal on Selected Areas in Communications, vol. 26, no. 1, pp. $38-51,2008$.

[30] T. W. M. Robert A. Monzingo, Introduction to Adaptive Arrays, 1st ed. John Wiley \& Sons, 2004.

[31] L. N. Trefethen and D. Bau, Numerical Linear Algebra. SIAM: Society for Industrial and Applied Mathematics.

[32] D. P. Palomar and e. Yonina C. Eldar, Convex Optimization in Signal Processing and Communications. Cambridge University Press, 2009.

[33] M. Grant and S. Boyd, "CVX: Matlab software for disciplined convex programming," http://cvxr.com/cvx, Sep. 2012.

[34] L. Fu, Y. J. Zhang, and J. Huang, "Energy efficient transmissions in MIMO cognitive radio networks," in Annual Conference on Information Sciences and Systems (CISS), 2012 46th, 2012, pp. 1-6.

[35] S. Boyd and L. Vandenberghe, Convex Optimization, 2004.

[36] J. R. Magnus and H. Neudecker, Matrix Differential Calculus with Applications in Statistics and Econometrics, ser. ISBN 0-471-986321, V. Barnett and N. A. C. Cressie, Eds. JOHN WILEY \& SONS, 2007.

[37] RFMD. (2013, Jun.) Website. [Online]. Available: http://www.rfmd.com/ 